\title{
Formulación y caracterización de bebidas nutricionales con base a zapallo y lactosuero, enriquecidas con avena y maracuyá
}

\section{(Formulation and characterization of a nutritional pumpkin- based whey beverages, enriched with oatmeal and passion fruit)}

\author{
Alex Valencia ${ }^{1}$, Liliana Acurio ${ }^{1}$, Lander Pérez ${ }^{1}$, Diego Salazar ${ }^{1}$, Verónica Tamayo $^{1}$
}

\section{Resumen:}

En este trabajo se determinó el efecto de la incorporación de avena (Avena sativa) y maracuyá (Passiflora edulis) en el contenido en fibra, calcio, hierro, fósforo y vitamina $A$ en bebidas formuladas a base de zapallo (Cucurbita máxima), leche y suero. Las bebidas sufrieron cambios significativos durante el tiempo de almacenamiento, el pH disminuyó mientras que la acidez mostró un ligero incremento. Las coordenadas de CIELAB $\left(L^{*}, a^{*}\right.$ y $\left.b^{*}\right)$ se ubicaron en el cuadrante 1 que indican una clara tendencia al color amarillo producto de la presencia de carotenoides provenientes del zapallo y la maracuyá. En base al análisis sensorial se seleccionó el mejor tratamiento, aquel que fue superior en 3 de los 4 parámetros evaluados. Esta bebida presentó además un comportamiento de fluido pseudoplástico con un índice de consistencia de 3,57 Pa.s.24 y con un tiempo de vida útil de 9 días.

Palabras clave: bebidas; zapallo; avena; maracuyá; leche; suero.

\begin{abstract}
:
In this work, the effect on the addition of oatmeal (Avena sativa) and passion fruit (Passiflora edulis) in the content of fiber, calcium, iron, phosphorus and $A$ vitamin in beverage formulation based on pumpkin (Cucurbita maxima), milk and whey, was determined. The beverages undergone significant changes during the storage time, the $\mathrm{pH}$ decreased as the acidity showed a slight increase. The coordinates of $\operatorname{CIELAB}\left(\mathrm{L}^{*}, a{ }^{*}\right.$ and $\left.b^{*}\right)$ were located in quadrant 1 indicating a clear tendency to yellow caused by the presence of carotenoids from the pumpkin and passion fruit. Based on the sensory analysis it was selected best treatment who was higher in 3 of the 4 parameters evaluated. This sample also presented a pseudoplastic behavior with a consistency index of 3.57 Pa.s ${ }^{0.24}$ and a shelf life of 9 days.
\end{abstract}

Keywords: beverages; pumpkin; oatmeal; passion fruit; milk; whey.

\footnotetext{
${ }^{1}$ Universidad Técnica de Ambato, Ambato - Ecuador ( \{avalencia, Ip.acurio, Iv.perez, dm.salazar, v.tamayo\} @uta.edu.ec )
} 


\section{Introducción}

El zapallo, la avena y la maracuyá son cultivos tradicionales en la región andina de América del Sur. Son ricos en aminoácidos, fibra y vitaminas además de ser reconocidos por sus propiedades beneficiosas para la salud. El zapallo (Cucurbita máxima) es una cucurbitácea cuya característica principal de la pulpa es su contenido de carotenoides. Algunos como $\beta$-caroteno y $\alpha$-caroteno, son precursores de la vitamina $A$, una vitamina soluble en grasa que es importante para el crecimiento y desarrollo normal del cuerpo humano (Rao \& Rao, 2007). La avena (Avena sativa), contiene $\beta$ glucanos los cuales presentan numerosas aplicaciones en la formulación de alimentos funcionales útiles para reducir el contenido de grasa y calorías (Lee et al., 2004b).

La maracuyá (Passiflora edulis), es una fruta muy apreciada por su sabor y aroma (Deliza et al., 2004), rica en carotenoides que a su vez son responsables de diversas funciones biológicas como la actividad antioxidante (Rosso \& Mercadante, 2007). Lo anterior permite establecer la posibilidad de desarrollar formulaciones de bebidas con base a zapallo, suero y leche, que además pueden ser enriquecidas con avena y maracuyá.

Por otro lado, los programas gubernamentales de alimentación de países en vías de desarrollo podrían verse favorecidos al disponer de un alimento con el aporte de proteínas de alto valor biológico de la leche (Salgado \& Restrepo, 2008), de bajo contenido en colesterol por la presencia de suero (Inda, 2000)., fibra de la avena y vitaminas y sabor de la maracuyá. Para tal fin, se requiere encontrar la mezcla óptima de ingredientes que permita generar las mejores características organolépticas, físicas, reológicas o químicas. Con base en lo anterior, el objetivo del trabajo fue desarrollar una bebida con base a zapallo, leche y suero, enriquecida con avena y maracuyá, destinada a niños en edad escolar.

\section{Metodología}

\section{Preparación de la bebida}

La bebida fue elaborada a partir de: zapallo, leche, suero, avena y maracuyá adquiridos en un supermercado de la ciudad de Ambato. La Tabla 1 muestra los niveles de zapallo, leche, suero, avena y maracuyá que se utilizan como límites para la aplicación del diseño experimental de mezclas (Statgraphics Centurión) y que permiten obtener las proporciones que se muestran en la Tabla 2.

Del zapallo se retiraron las semillas y la cáscara y se llevó a ebullición durante 60 minutos. El suero de leche, donado por una quesería local y obtenido por fermentación enzimática, fue pasteurizado por separado a $60{ }^{\circ} \mathrm{C}$ por $30 \mathrm{~min}$, transcurrido ese tiempo se incorporó hojuelas de avena, leche, pulpa de maracuyá y se ajustó los sólidos solubles a $15^{\circ}$ Brix, finalmente todos los 
ingredientes se licuaron y la mezcla se sometió a ebullición $\left(92{ }^{\circ} \mathrm{C}\right)$ por 15 min. Las muestras fueron envasadas en recipientes de vidrio y almacenadas en refrigeración a $4{ }^{\circ} \mathrm{C}$.

Tabla 1. Niveles de los componentes para la aplicación el diseño experimental de mezclas para la elaboración de bebidas con base a zapallo y lactosuero

\begin{tabular}{lccc}
\hline \multirow{2}{*}{ Componentes } & \multicolumn{2}{c}{ Niveles } & Unidades \\
\cline { 2 - 3 } A: Leche & Bajo & Alto & \\
B: Suero & 30 & 40 & $\%$ \\
C: Zapallo & 25 & 35 & $\%$ \\
D: Avena & 30 & 40 & $\%$ \\
E: Maracuyá & 2 & 4 & $\%$ \\
\hline
\end{tabular}

Tabla 2. Concentración de los diferentes componentes utilizados para la elaboración de bebidas con base a zapallo y lactosuero

\begin{tabular}{cccccc}
\hline \multirow{2}{*}{ Nomenclatura } & \multicolumn{5}{c}{ Componente (\%) } \\
\cline { 2 - 6 } & Leche & Suero & Zapallo & Avena & Maracuyá \\
\hline 40L25S30Z2A3M & 40 & 25 & 30 & 2 & 3 \\
30L35S30Z2A3M & 30 & 35 & 30 & 2 & 3 \\
30L25S40Z2A3M & 30 & 25 & 40 & 2 & 3 \\
38L25S30Z4A3M & 38 & 25 & 30 & 4 & 3 \\
30L33S30Z4A3M & 30 & 33 & 30 & 4 & 3 \\
30L25S38Z4A3M & 30 & 25 & 38 & 4 & 3 \\
37L25S30Z2A6M & 37 & 25 & 30 & 2 & 6 \\
30L32S30Z2A6M & 30 & 32 & 30 & 2 & 6 \\
30L25S37Z2A6M & 30 & 25 & 37 & 2 & 6 \\
35L25S30Z4A6M & 35 & 25 & 30 & 4 & 6 \\
30L30S30Z4A6M & 30 & 30 & 30 & 4 & 6 \\
30L25S35Z4A6M & 30 & 25 & 35 & 4 & 6 \\
\hline
\end{tabular}

L: leche; S: suero; Z: zambo; A: avena y M: maracuyá

\section{Propiedades fisicoquímicas}

Se determinó la variación de $\mathrm{pH}$ y acidez desde el momento de la elaboración hasta 9 días de almacenamiento en condiciones de refrigeración $\left(4^{\circ} \mathrm{C} \pm 2{ }^{\circ} \mathrm{C}\right)$. El pH se determinó mediante un potenciómetro HANNA HI 9126 (Rhode Island, EE.UU.). La acidez fue determinada por titulación con $\mathrm{NaOH} 0,1 \mathrm{~N}$, utilizando como indicador fenolftaleína acorde a la metodología descrita en la AOAC 947.05. Cada determinación se realizó al menos por triplicado.

\section{Determinación de color}

Después de la pasteurización se midieron los parámetros de color, L* (luminosidad), a* (rojo/verde) b* (amarillo/azul), índice de amarillo y brillo con un colorímetro Konica Minolta CM 3500 d (Konica Minolta, España), calibrado con el iluminador D65 (luz natural) y el observador estándar D10. La coordenada polar chroma o saturación $\mathrm{C}^{*}$, puede ser calculada a partir de la 
expresión $C^{*}=\sqrt{ }\left(a^{* 2}+b^{* 2}\right)$. Las medidas se realizaron en 5 zonas diferentes de la muestra y cada valor fue el promedio de las medidas de al menos 3 muestras diferentes

\section{Análisis reológico}

Se utilizó un viscosímetro rotacional Quimis Q860M21 (Sao Paulo, Brasil) de medición relativa y un vaso de precipitados de $500 \mathrm{~mL}$, en el cual se introdujo la muestra a analizar a $4{ }^{\circ} \mathrm{C} \pm 2{ }^{\circ} \mathrm{C}$, por triplicado. Se preparó el equipo colocando el rotor \#1 ya que se adaptó óptimamente a las características del producto estudiado. Se procedió a realizar las lecturas de viscosidad respectivas para cada muestra, siguiendo las directrices de Steffe (1996) y Schramm (1994). A partir de los datos de viscosidad obtenidos a diferentes gradientes de cizalladura se determinó el tipo de comportamiento reológico.

\section{Análisis sensorial}

Para el análisis sensorial, se evaluaron 12 formulaciones de bebidas (Tabla 2) y un control (marca comercial), para el efecto se consideraron los atributos de color, aroma, sabor y aceptabilidad. El ensayo se llevó a cabo con un panel formado por 26 niños en edad escolar de la Unidad Educativa Gabriela Mistral (Ambato-Ecuador), de entre 8 a 10 años. Se aplicó un diseño de bloques ajustados (Cocchran, 1974). Cada catador recibió tres muestras de $100 \mathrm{ml}$ cada una. El tipo de ficha que se adoptó fue gráfica con caras de expresión de agrado o desagrado, es decir, una escala hedónica de 1 a 5 , siendo 1 la puntuación más baja y 5 la más alta, apropiada para niños que evalúan el producto.

\section{Análisis proximal}

A la bebida determinada como mejor tratamiento, se analizó humedad, siguiendo la metodología descrita en la AOAC 19 927.05; cenizas, según el método de calcinación (AOAC 923.03); contenido de proteína según el método AOAC 2001.11; grasa con el método Soxlet (AOAC 2033.06); fibra dietética según lo descrito en la AOAC 985.29; carbohidratos totales por diferencia, energía metabolizante mediante el porcentaje de kilocalorías que cada nutriente energético tiene en relación al total.

Además, se analizó el perfil de minerales, hierro por el método AOAC 944.02; calcio según el método APHA 4500-CA; potasio basado en el método APHA 3500-K; fósforo por espectrofotometría mediante la formación de un complejo coloreado de fosfomolibdato $\left[\left(\mathrm{MoO}_{2} 4 \mathrm{MoO}_{3}\right) 2 \mathrm{H}_{3} \mathrm{PO}_{4}\right]$ que en presencia de ácido ascórbico se reduce a azul de molibdeno y cuya absorbancia se lee a una longitud de onda de $660 \mathrm{~nm}$. Y finalmente se analizó el contenido de vitamina A según el método AOAC 992.06. Cada determinación se realizó al menos por triplicado. 


\section{Vida útil}

La bebida se almacenó en envases de vidrio de $240 \mathrm{ml}$ en refrigeración $\left(4^{\circ} \mathrm{C} \pm 2{ }^{\circ} \mathrm{C}\right)$ durante 0,2 , 5, 7, 9 y 12 días. Durante este período se realizaron recuentos de aerobios mesófilos totales según lo descrito en la norma técnica ecuatoriana INEN 1529-5:2006.

\section{Diseño experimental}

Las concentraciones de los componentes, establecidas en las diferentes formulaciones (codificadas en composición y fracción de 0 a 100, según el intervalo alto o bajo): leche 30-40\%, suero $25-35 \%$, zapallo $30-40 \%$, avena $2-4 \%$ y maracuyá $3-6 \%$ y los resultados de los análisis realizados, fueron tabulados utilizando la herramienta Statgraphics (Virginia, EE.UU.). Las diferencias se evaluaron mediante la prueba de Tukey HSD al 95\% de confianza

\section{Resultados y discusión}

En la Figura 1 se muestran las variaciones de $\mathrm{pH}$ de las bebidas durante el almacenamiento (9 días), de los datos obtenidos se observa que los porcentajes que corresponden a los niveles altos de leche y maracuyá influyen en la variación del pH.

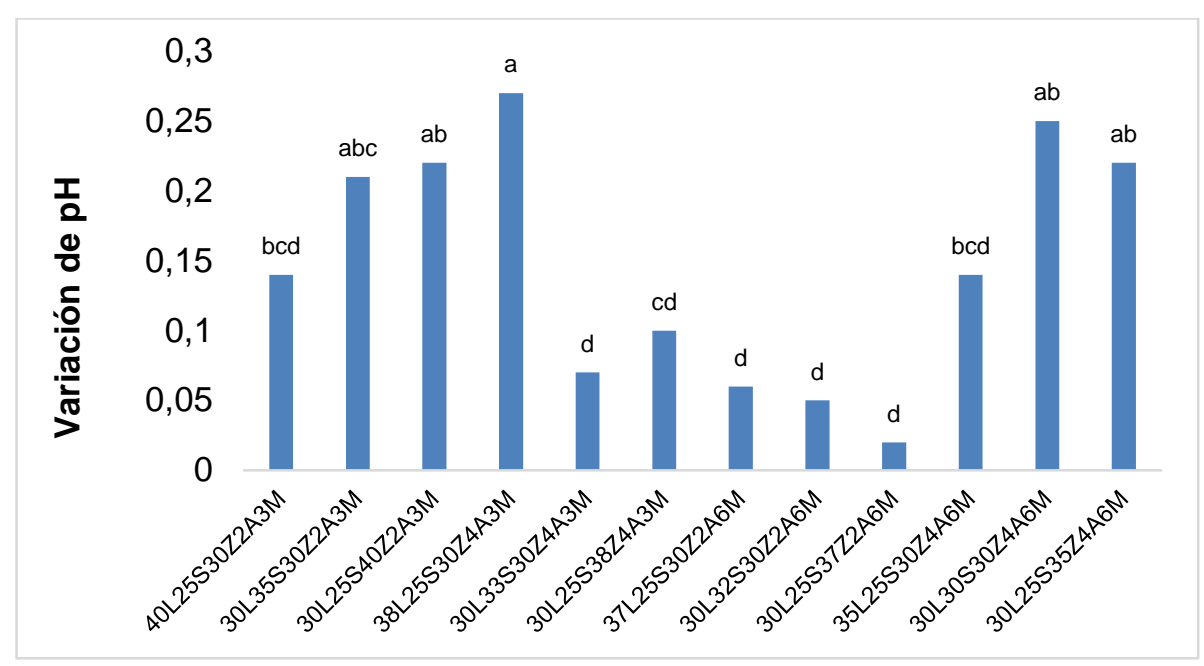

Figura 1. Variación de pH durante el almacenamiento

La variación de acidez expresada en porcentaje de ácido láctico mostró un incremento en los tratamientos, posiblemente debido al efecto conjunto de los componentes en la mezcla (Figura 2). Según De Paula et al. (2014), la disminución de pH se debe a la fermentación bacteriana de los azúcares presentes (glucosa, galactosa y lactosa), lo cual podría estar asociado con las diferencias obtenidas en el $\mathrm{pH}$ de las bebidas. 


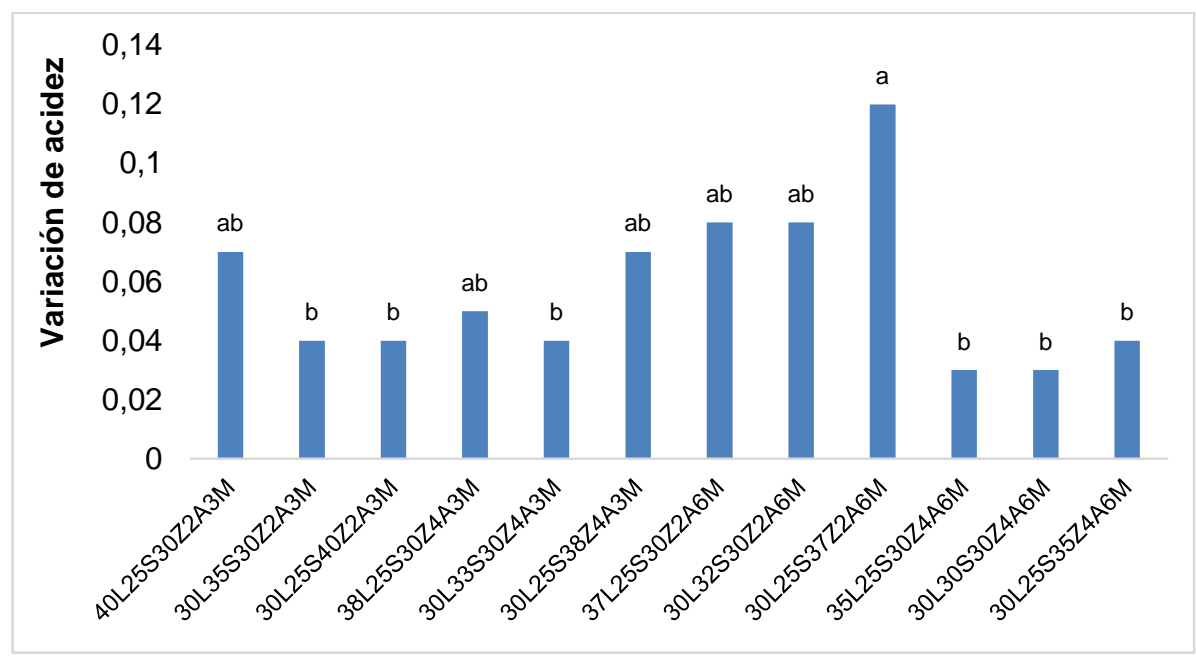

Figura 2. Variación de acidez durante el almacenamiento

Los resultados del análisis de color $L^{*}, a^{*}, b^{*}$, índice de amarillo y brillo se presentan en la Figura 3. La luminosidad ( $L$ ) fue similar en todas las muestras, no se encontraron diferencias significativas entre muestras. Con respecto a los valores de $a^{*} \mathrm{y} \mathrm{b}^{*}$ si se obtuvieron diferencias significativas $(\mathrm{P}<0.05)$, muchas de ellas asociadas principalmente a la diferente concentración de maracuyá y zapallo en la formulación.

Los valores del índice de amarillo mostraron diferencias significativas $(P<0.05)$ en todas las muestras. La bebida 30L25S40Z2A3 (30\% leche, 25\% suero, 40\% zapallo, $2 \%$ avena, y $3 \%$ maracuyá) presentó, de entre todas las bebidas, un mayor índice de amarillo, una mayor tendencia al amarillo ( $\left.{ }^{*} \mathrm{~b}\right)$ y en un color vívido por un $\mathrm{C}^{*}$ alto en relación al resto de las muestras. Estos resultados se pueden atribuir al alto contenido de zapallo en comparación con las demás bebidas. Resultados similares fueron observados por Gonçalves et al. (2007), quienes explicaron que la disminución en los valores de CIEL $a^{*}, b^{*}$ y $C^{*}$ y las variaciones en el color de calabaza cocida están relacionadas a la degradación de los carotenoides. 

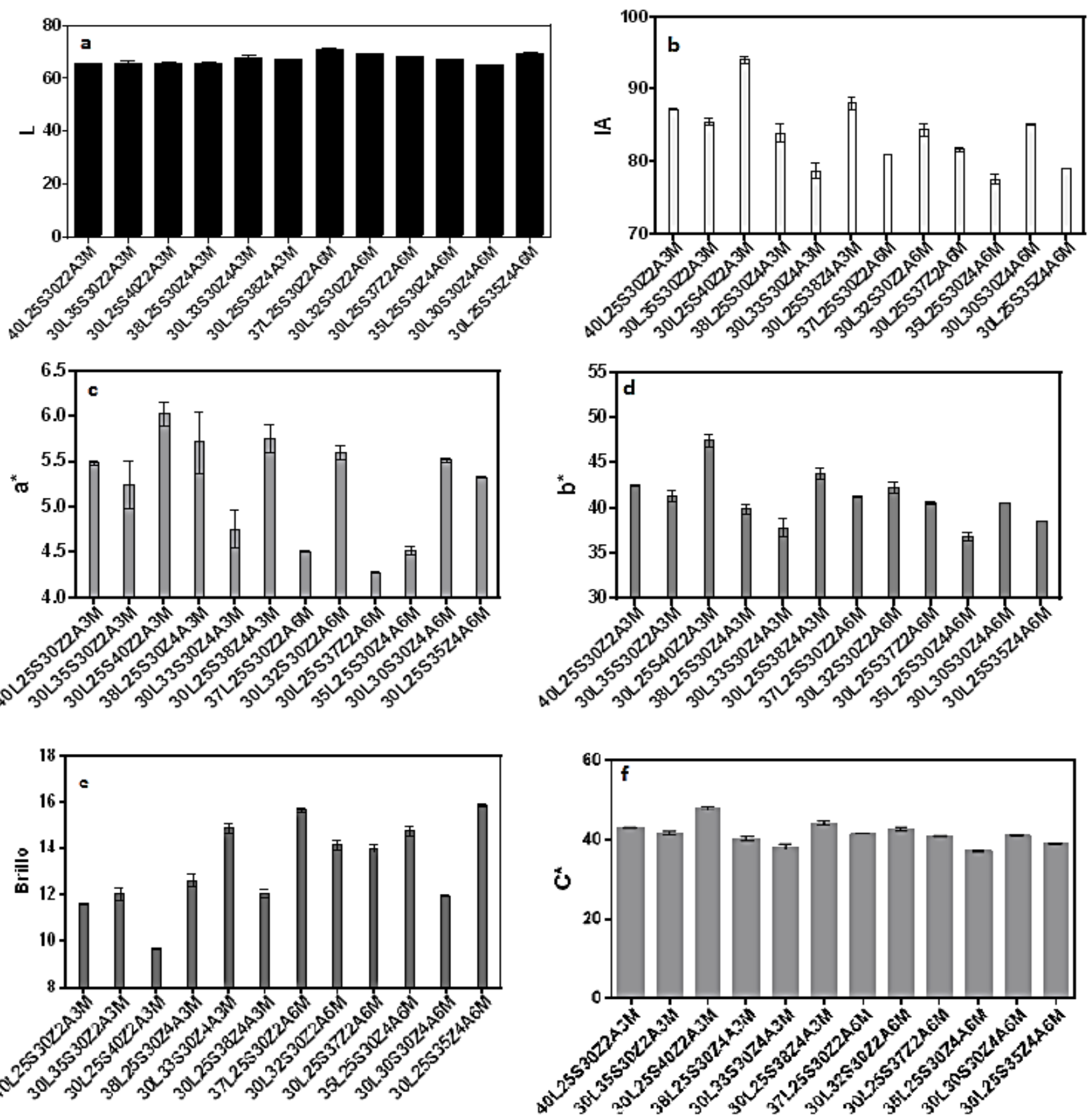

Figura 3. Parámetros de color $L(a), a^{*}(c), b^{*}(d)$, índice de amarillo (b), brillo (e) y saturación o Croma ( $\left.C^{*}\right)$, de las diferentes bebidas. L: leche; S: suero; Z: zambo; A: avena y M: maracuyá.

En cuanto al comportamiento reológico, se observó mayor índice de consistencia en los tratamientos que contienen: $30 \%$ leche, $25 \%$ suero, $35 \%$ zapallo, $4 \%$ avena, $6 \%$ maracuyá y $30 \%$ leche, $25 \%$ suero, 38\% zapallo, $4 \%$ avena, 3\% maracuyá (Tabla 3). Evidentemente la leche y el suero por su estructura fluida disminuyen la viscosidad de la bebida, mientras que la presencia de avena aumentan la consistencia del producto. La avena ejerce un efecto espesante que se debe a la alta cantidad de hidratos de carbono $(60 \mathrm{~g} / 100 \mathrm{~g})$ constituidos principalmente por almidón (Sandhu et al.2015), y a la fibra dietética soluble presente en cantidades de 1,8 a 7,5 g/100 g (Bhatty, 1992). La fibra soluble está compuesta principalmente por $\beta$-glucano, que presenta buena retención de agua y proporciona estabilidad a la bebida, éste efecto es aprovechado 
industrialmente para mejorar las propiedades texturales y reológicas de diferentes alimentos (Lee et al., 2002; Rosell et al., 2001; Sandhu et al.2015).

Al aplicar la Ley de la Potencia se observó que el índice de comportamiento al flujo (n) presenta valores menores a uno, confirmando que la bebida tiene un comportamiento no newtoniano del tipo pseudoplástico.

Tabla 3. Valores de índice de comportamiento de flujo (n) e índice de consistencia (K) $\left[\right.$ Pa.s $\left.{ }^{n}\right]$ para cada bebida.

\begin{tabular}{ccc}
\hline Muestra & $\mathbf{K}\left[\mathbf{P a} . \mathbf{s}^{\mathbf{n}}\right]$ & $\mathbf{n}$ \\
\hline 40L25S30Z2A3M & $11,17 \pm 1,83$ & $0,25 \pm 0,04$ \\
30L35S30Z2A3M & $9,71 \pm 0,58$ & $0,25 \pm 0,05$ \\
30L25S40Z2A3M & $7,97 \pm 0,48$ & $0,26 \pm 0,08$ \\
38L25S30Z4A3M & $5,63 \pm 0,27$ & $0,23 \pm 0,02$ \\
30L33S30Z4A3M & $9,04 \pm 0,54$ & $0,40 \pm 0,02$ \\
30L25S38Z4A3M & $16,13 \pm 0,80$ & $0,42 \pm 0,03$ \\
37L25S30Z2A6M & $5,07 \pm 0,18$ & $0,42 \pm 0,01$ \\
30L32S30Z2A6M & $7,95 \pm 0,49$ & $0,33 \pm 0,02$ \\
30L25S37Z2A6M & $5,48 \pm 0,32$ & $0,33 \pm 0,08$ \\
35L25S30Z4A6M & $3,57 \pm 0,30$ & $0,24 \pm 0,02$ \\
30L30S30Z4A6M & $9,03 \pm 0,53$ & $0,24 \pm 0,04$ \\
30L25S35Z4A6M & $16,86 \pm 1,14$ & $0,32 \pm 0,02$ \\
\hline
\end{tabular}

L: leche; S: suero; Z: zapallo; A: avena y M: maracuyá

De la evaluación sensorial de las bebidas, se pudo establecer a un 95\% de nivel de confianza, que existe diferencias significativas entre tratamientos, siendo el de mejor puntuación aquel que contiene 35\% leche, 25\% suero, 30\% zapallo, 4\% avena, y 6\% maracuyá (35L25S30Z4A6M) (Figura 4). Esta bebida obtuvo las puntuaciones más altas en 3 de los 4 atributos (aroma, sabor y aceptabilidad).

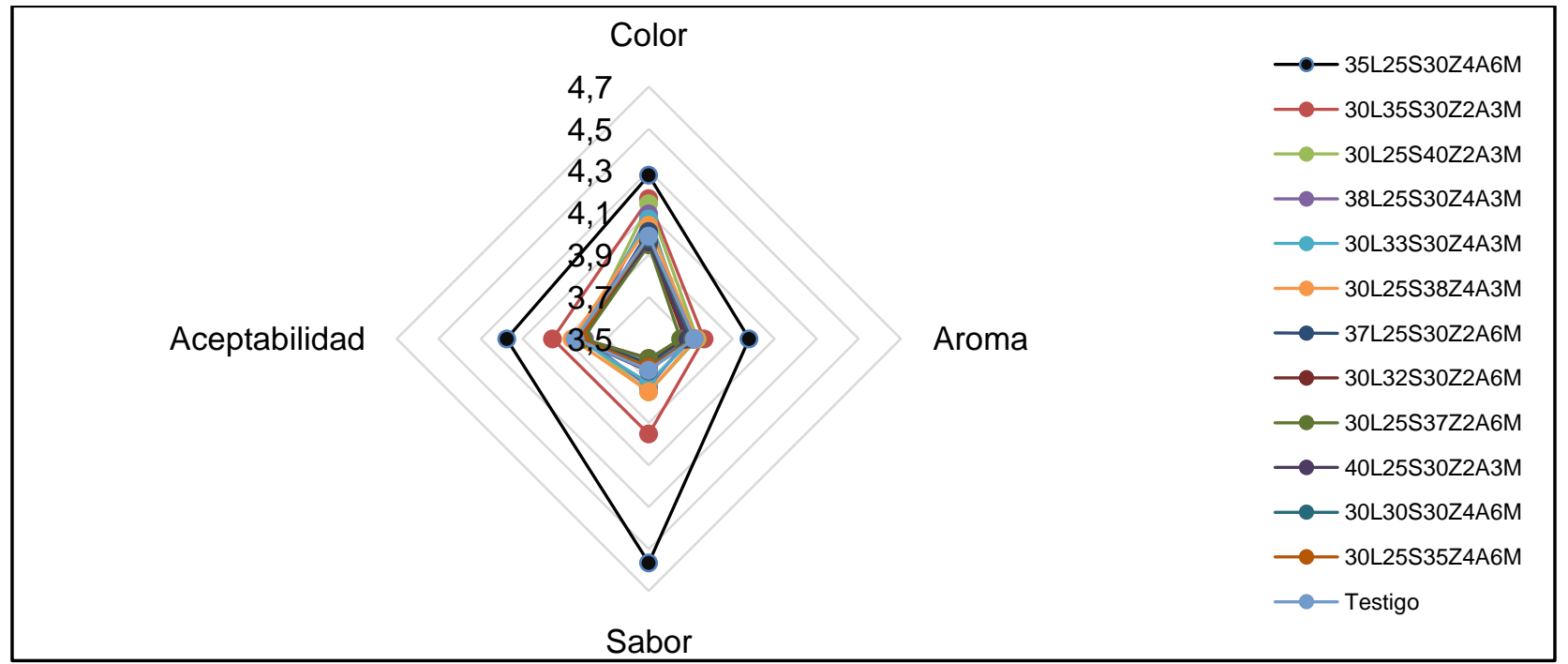

Figura 4. Resultados de la evaluación sensorial de las bebidas obtenidas. L: leche; S: suero; Z: zapallo; A: avena y M: maracuyá. 
En el diseño de mezclas los factores que intervienen son las proporciones de los componentes de la mezcla y las respuestas a optimizar son función de esas proporciones, con respecto al total y no dependen de la cantidad de cada componente (Solís et al., 2008). El analisis de varianza permitió establecer un efecto altamente significativo (valor- $p=0,000)$ en el modelo lineal que permite medir el efecto de las variables (leche, suero, zapallo, avena y maracuyá) sobre la apreciación óptima del pH, acidez, índice de amarillo e índice de consistencia.

Los parámetros derivados del diseño presentan características diversas, el pH se ajusta a una relación lineal (Ec. 1).

$$
p H=4,88 L+5 S+4,84 Z+6,85 A+4,46 M
$$

Ecuación 1

La acidez se ajusta a la Ecuación 2.

$$
\text { Acidez }=0,16 L+0,15 S+0,16 Z-0,04 A+0,3 M
$$

Ecuación 2

El índice de amarillo a la Ecuación 3.

$$
\text { Índice de amarillo }=86,4 L+86,5 S+91,18 Z+70,34 A+72,01 M
$$

Ecuación 3

El brillo la Ecuación 4.

$$
\text { Brillo }=12,19 L+12,08 S+10,93 Z+15,81 A+19,27 M
$$

Ecuación 4

Y el índice de consistencia a la Ecuación 5.

$$
\text { Índice de consistencia }=6,33 L+8,84 S+11,42 Z+19,58 A+2,34 M
$$

Ecuación 5

Donde L, S, Z, A y M corresponden a leche, suero, zapallo, avena y maracuyá respectivamente.

La Tabla 4 presenta la ecuaciones y el tiempo de vida útil (9 días), obtenido mediante recuento en placa de aerobios mesófilos totales con base al límite máximo de $50000 \mathrm{ufc} / \mathrm{cm}^{3}$ presentado en la Norma NTE INEN 2564:201. El desarrollo microbiano mostrado corrobora las tendencias de disminución de $\mathrm{pH}$ e incremento de acidez discutidos anteriormente. Salazar \& Sepúlveda (2005), documentaron 18 días de vida de anaquel en una bebida nutritiva de máchica y leche, del mismo modo Choto (2012) obtuvo 21 días en una bebida con suero y avena. Este periodo depende de muchas variables en donde se incluyen tanto el producto como las condiciones ambientales y el envase.

Tabla 4. Ecuación de regresión y tiempo de vida útil a partir del recuento microbiológico (ufc/ml)

\begin{tabular}{cccc}
\hline Muestra & Regresión & $\mathbf{R}^{2}$ & $\begin{array}{c}\text { Tiempo de vida } \\
\text { útil (días) }\end{array}$ \\
\hline 35L25S30Z4A6M & $\mathrm{LnC}($ ufc $)=0,715(\mathrm{t})+4,75$ & 0,99 & 9 \\
\hline
\end{tabular}


En la Tabla 5, se presentan los resultados del análisis proximal de donde, el consumo de 100 gramos de esta bebida aportarían al organismo $57 \mathrm{kcal}$ debido principalmente al 2,03\% de proteína, $1,46 \%$ de grasa y $9 \%$ de carbohidratos. La bebida desarrollada posee $4,45 \%$ de fibra dietética y $0,63 \%$ de cenizas dentro de la que se encuentran micro elementos, obteniendo un alto contenido de calcio, hierro, fosforo y vitamina $A$, este último se debe al elevado contenido de $\beta$ carotenos aportados por el zapallo y maracuyá. La bebida, por sus características nutricionales superiores, puede ser muy útil en programas gubernamentales, bares de instituciones educativas, entre otras, y así contribuir en las etapas de desarrollo y crecimiento de los niños en edad escolar. (8-10 años). Las necesidades de la población infantil están condicionadas por el crecimiento del cuerpo y el desarrollo de los huesos, dientes, músculos, entre otros. Los niños requieren energía en forma de hidratos de carbono y grasa, proteínas de alto valor biológico, minerales como el hierro, calcio y vitaminas (Cervera et al., 1992).

Tabla 5. Contenido nutricional en $100 \mathrm{~g}$ de bebida que contiene $35 \%$ leche,

\begin{tabular}{lcc}
\multicolumn{2}{c}{ Ens suero, 30\% zapallo, 4\% avena, y $6 \%$ maracuyá } & (35L25S30Z4A6M). \\
\hline Humedad & Unidades & Resultados \\
Cenizas & $\%$ & 82 \\
Proteínas & $\%$ & 0,63 \\
Grasa & $\%\left(\mathrm{~N}^{*} 6,25\right)$ & 2,03 \\
Fibra dietética Total & $\%$ & 1,46 \\
Carbohidratos Totales & $\%$ & 4,45 \\
Energía & $\%$ & 9 \\
Hierro & $\mathrm{kcal} / 100 \mathrm{~g}$ & 57 \\
Calcio & $\mathrm{mg} / 100 \mathrm{~g}$ & 1,958 \\
Potasio & $\mathrm{mg} / 100 \mathrm{~g}$ & 136,17 \\
Fósforo & $\mathrm{mg} / 100 \mathrm{~g}$ & 77,88 \\
Vitamina A & $\mathrm{mg} / 100 \mathrm{~g}$ & 109,17 \\
\hline
\end{tabular}

\section{Conclusiones}

La investigación permitió desarrollar una nueva alternativa tecnológica para elaborar una bebida utilizando como materia prima zapallo, avena y maracuyá. Además se identificó la mejor formulación como aquella que contiene $35 \%$ leche, $25 \%$ suero, $30 \%$ zapallo, $4 \%$ avena, y $6 \%$ maracuyá lo cual fue ratificado de acuerdo al mejor criterio de los niños de 8 a 10 años.

La bebida nutricional contiene cantidades significativas de proteína, fibra dietética, calcio, hierro, fósforo y vitamina $\mathrm{A}$. Con un tiempo de vida útil de 9 días en condiciones de refrigeración $\left(4{ }^{\circ} \mathrm{C} \pm\right.$ 2), lo que la convierte en una bebida con una alto contenido nutricional. 


\section{Bibliografía}

Bhatty, R. S. (1992). Total and extractable $\beta$-glucan contents of oats and their relationship to viscosity. Journal of Cereal Science, 15(2), 185-192. doi: http://dx.doi.org/10.1016/S07335210(09)80070-2

Cervera, P., Clapes, J., \& Rigolfas, R. (1992). Alimentación y Dietoterapia. Barcelona-España: Grupo Editorial Interamericana Mc Graw-Hill.

Choto, E. (2012). Diseño del proceso de elaboración de una bebida nutritiva de machica y leche para la molinera San Luis. Tesis de grado de Ingeniería Química. Facultad de CienciasESPOCH, Riobamba-Ecuador.

Cocchran, W. (1974). Técnicas de muestreo (Cuarta ed.). México, España, Argentina y Chile: Continental, S. A.

De Paula, C., Martinez, A. P., \& Nuñez, M. (2014). Evaluación sensorial de una bebida deslactosada y Fermentada a partir de Lactosuero adicionada con pulpa de maracuyá. Congreso Iberamericano de Ingeniería de Alimentos (CIBIA 9), 93-100.

Deliza, R., MacFie, H., \& Hedderley, D. (2004). The consumer sensory perception of passion-fruit juice using free-choice profiling. Journal of Sensory Studies, 19, 557-587.

Goncalves, E.; Pinheiro, J.; Abreu, M.; Brandao, T., \& Silva, F. (2007). Modelling the kinetics of peroxidase inactivation, colour and texture changes of pumpkin (Cucurbita maxima L.) during blanching. Journal of Food Engineering. 81 (4): 693-701.

Inda, A. (2000). Optimización de Rendimiento y Aseguramiento de Inocuidad en la Industria de la Quesería. México: Organización de los Estados Americanos OEA.

Lee, M., Baek, M., Cha, D., Park, H., \& Lim, S. (2002a). Freeze-thaw stabilization of sweet potato starch gel by polysaccharide gums. Food Hydrocolloids, 16, 345-352.

Lee, S., Inglett, G., \& Carriere, C. (2004b). Effect of nutrim oat bran and flaxseed on rheological properties of cakes. Cereal Chemistry, 81, 637-642.

Rao, A., \& Rao, L. (2007). Carotenoids and human health. Pharm, 207-206.

Rosell, C., Rojas, J., \& De Barber, C. B. (2001). Influence of hydrocolloids on dough rheology and bread quality. Food Hydrocolloids, 15(1), 75-81. 
Rosso, V., \& Mercadante, A. (2007). Identification and quantification of carotenoids, by HPLCPDA-MS/MS, from Amazonian fruits. Journal of Agricultural and Food Chemistry, 55, 50625072.

Solís, N., Márquez, J., Cervantes, E., \& Armijo, J. (2008). Diseño de experimentos. México: UANE

Salazar, B. M., \& Sepúlveda, J. (2005). Viabilidad de un aislado nativo de Lactobacillus brevis en una bebida láctea Fermentada. Scielo, 350-353.

Salgado, C., \& Restrepo, M. (2008). Importancia de los Lácteos en la Nutrición. Revista I Alimentos, 38-40.

Sandhu, K. S., Godara, P., Kaur, M., \& Punia, S (2015). Effect of toasting on physical, functional and antioxidant properties of flour from oat (Avena sativa L.) cultivars. Journal of the Saudi Society of Agricultural Sciences. doi: http://dx.doi.org/10.1016/j.jssas.2015.06.004

Schramm, G. (2000). A Practical Approach to Rheology and Rheometry. 2nd Ed. (R. Steinbrüggen, Trans.): Gebrueder HAAKE GmbH, Karlsruhe (RFA).

Steffe, J. (1996). Rheological Methods in Food Process Engineering. East Lansing, Michigan (USA): Freeman Press 